\title{
Identification of short-form RON as a novel intrinsic resistance mechanism for anti-MET therapy in MET-positive gastric cancer
}

\author{
Zheng Wu ${ }^{1,2, *}$, Zhe Zhang ${ }^{1,2, *}$, Xiaoxiao Ge ${ }^{1,2}$, Ying Lin ${ }^{1,2}$, Congqi Dai ${ }^{1,2}$, Jinjia Chang ${ }^{1,2}$, \\ Xinyang Liu ${ }^{1,2}$, Ruixuan Geng ${ }^{1,2}$, Chenchen Wang ${ }^{1,2}$, Huan Chen ${ }^{2,3}$, Menghong Sun ${ }^{2,3}$, \\ Weijian Guo ${ }^{1,2}$, Jin $\mathbf{L i}^{1,2}$ \\ ${ }^{1}$ Department of Medical Oncology, Fudan University Shanghai Cancer Center, Shanghai 200032, China \\ ${ }^{2}$ Department of Oncology, Shanghai Medical College, Fudan University, Shanghai 200032, China \\ ${ }^{3}$ Department of Pathology, Fudan University Shanghai Cancer Center, Shanghai 200032, China \\ *These authors have contributed equally to this work \\ Correspondence to: \\ Jin Li, e-mail: fudanlijin@163.com \\ Keywords: drug resistance, RON, MET, gastric cancer, targeted therapy \\ Received: April 20, $2015 \quad$ Accepted: September 22, $2015 \quad$ Published: October 26, 2015
}

\section{ABSTRACT}

Despite the promising results from initial studies, there are significant limitations in the application of MET-targeted therapy in gastric cancer. Intrinsic resistance is one of the major obstacles. The aim of this study is to identify the responsible receptor tyrosine kinases (RTKs) that determine the unresponsiveness of MET inhibitor in MET-positive gastric cancer. through an RNA-interference-based functional screen targeting most human RTKs, we identified that activation of the fibroblast growth factor receptor 2 (FGFR2) and recepteur d'origine nantais (RON) pathways attenuated MET inhibitor-induced suppression of cell proliferation and migration. Notably, in the two forms of RON pathway activation, only upregulation of short-form RON (sf-RON), but not stimulation of full length RON with macrophage stimulating protein, conferred MET inhibitor resistance in vitro and in vivo. Furthermore, the profile of the gastric cancer samples observed that sf-RON was frequently upregulated in METpositive gastric cancer. Our findings indicate that activation of the sf-RON signaling pathway represents a novel mechanism underlying MET inhibitor unresponsiveness. A combination strategy with drugs targeting both RON and MET pathways is believed to improve the efficacy of MET-targeted therapy.

\section{INTRODUCTION}

MET, as a member of receptor tyrosine kinase (RTK) family, plays a causal role in tumor cell survival, growth, angiogenesis and metastasis [1]. MET gene amplification has been reported in 1.5 to $10.2 \%$ of gastric cancers (GCs), and overexpression of MET can be detected in more than $20 \%$ of GC samples [2-6]. Excessive activation of the hepatocyte growth factor/scatter factor (HGF/SF)-MET axis is considered to correlate with poor prognosis and drug resistance in various human cancers, including GC $[1,2,7,8]$. Thus, anti-MET has emerged as an attractive strategy to treat patients with $\mathrm{GC}$ that harbors dysregulated HGF/SF-MET signaling. During the past decade, monoclonal antibodies and small-molecule tyrosine kinase inhibitors targeting the HGF/SF-MET pathway have been evaluated in preclinical experiments and clinical trials [9]. Initial results from most studies suggest that antiMET therapy is able to improve overall survival (OS) and progression-free survival (PFS) in several cancer types [9]. Recently, a double-blind and randomized phase 2 clinical trial showed that the addition of rilotumumab, a fully human monoclonal antibody of HGF, to chemotherapy improved the prognosis in patients with GC or esophagogastric junction cancer, especially in METpositive $(\mathrm{MET}+$ ) subgroup. However, in MET+ subgroup, the objective response rate to the treatment of rilotumumab plus chemotherapy was only 50\% [10]. Moreover, clinical trials studying the efficacy of MET-targeted tyrosine kinase inhibitors (TKIs) as monotherapy in patients with 
metastatic gastric adenocarcinoma only showed minimal efficacy, even in MET-amplified subgroup [11, 12]. Limited response rate and minimal efficacy suggested that a proportion of patients with MET+ stomach tumors may be insensitive to MET targeted therapy. Intrinsic resistance to MET inhibitors emerges as a major limitation in the application of MET-targeted therapy in gastric cancer.

In order to improve the efficacy of METtargeted therapy, understanding the potential molecular mechanisms of intrinsic resistance to MET inhibitors is imperative. Activation of compensatory pathways is recognized as an important sign of resistance to targeted therapies $[13,14]$. Despite inhibition of the drug targets, the presence of bypass RTKs that maintain the activation of downstream signaling pathways results in failure of targeted therapies [15]. In this study, we conducted a functional screening with a small interfering RNA (siRNA) library targeting most human RTKs, to identify bypass tracks that affect the responsiveness of MET+ GC to anti-MET agents. The results of screening and subsequent validation revealed that activation of fibroblast growth factor receptor 2 (FGFR2) and recepteur d'origine nantais (RON) pathways attenuated MET inhibitorinduced suppression of cell proliferation and migration. RON pathway was identified to promote resistance to antiMET agents for the first time. As another member of the MET proto-oncogene family [16], RON has been found to be activated aberrantly in various malignances, including GC [17], and is closely related to MET [1, 18]. Two transcripts of this gene coding a full-length RON (fl-RON) and a short-form RON (SF-RON) have been detected in GC tissues [19]. Here, we found that in the two forms of RON pathway activation, upregulation of sf-RON, but not stimulation of $\mathrm{f}-\mathrm{RON}$ with macrophage stimulating protein (MSP), conferred MET inhibitor resistance. We also found that sf-RON was up-regulated in MET+ GC. On the basis of these findings, MET/RON dual inhibition might be necessary for treating MET + GC patients with RON pathway activation.

\section{RESULTS}

\section{siRNA screening identifies RTKs influencing the sensitivity of GC cells to PF}

Firstly, to identify RTKs whose knockdown selectively sensitizes GC cells to MET inhibitor, a highly selective MET inhibitor PF and a MET-addicted GC cell line MKN-45 were chosen. This cell line is deemed to harbor MET gene amplification and stably over express MET [20]. Our results indicated that MET+ MKN-45 cells were much more responsive to PF than the other METGC cell lines, with an IC50 of $0.018 \mu \mathrm{M}$ (Supplementary Figure S1A and S1B). Then, MKN-45 cells were transfected with a siRNA library targeting 60 human RTK genes, with each gene targeted by 4 individual siRNAs. The combined effect of siRNA and PF was assessed using the SI. As mentioned previously [21], positive SI scores represented the sensitizing effect. All SI scores of 240 siRNA hits were ranked (Figure 1A), and RTKs with scores in the upper quartile were extracted for the subsequent analysis.

To avoid off-target effects, those genes with only one SI score in the first quarter were rejected. The remaining 16 candidates were re-assessed through cell proliferation inhibition assay. As a result, only knockdown of FGFR2, RON, Eph receptors A1 (EPHA1), and receptor-like tyrosine kinase (RYK), but not the other 12 RTKs significantly enhanced the effectiveness of PF in MKN-45 cells (Figure 1B and Supplementary Figure S2). The synergistic effect of silencing these 4 RTKs with $\mathrm{PF}$ was further confirmed in another MET-addicted GC cell line GTL-16 (Figure 1C). Together, these results suggested that multiple RTK pathways tend to influence the responsiveness of GC cells to MET inhibiton.

\section{B-FGF induced FGFR2 activation attenuates PF-induced growth inhibition and motility suppression}

In order to verify whether activation of EPHA1, RON, RYK, or FGFR2 pathways would contribute to PF resistance, we treated MKN-45 cells with PF $(0-0.02 \mathrm{uM})$ and corresponding ligands of each receptor for 10 days. The colony formation assay revealed that b-FGF-induced activation of FGFR2 resulted in an obvious increase in PF-resistant cell clones (Figure 2A). Conversely, no detectable disparity was observed between the control group and the other 3 ligand-treated groups (Figure 3A and Supplementary Figure S3A). We next confirmed the contribution of FGFR2 to PF resistance in GTL-16 cells. And the addition of AZD4547, an FGFR inhibitor [22], to $\mathrm{PF} / \mathrm{b}$-FGF treatment restored $\mathrm{PF}$-mediated proliferation inhibition (Figure 2B).

The activated MET signaling pathway not only promotes tumor cells proliferation, but also facilitates cell migration and invasion, which contributes to tumor metastasis [23-25]. Inhibition of MET through TKI or RNA interference was able to reduce lymph nodes or distant metastasis [26, 27]. By using transwell migration assay, we confirmed that PF markedly suppressed GTL-16 cell motility. Although treatment of b-FGF $(50 \mathrm{ng} / \mathrm{mL})$ alone could not enhance cell migration, b-FGF was able to nullify the motility suppression induced by PF. AZD4547 could neutralize the resistance to PF induced by b-FGF (Figure 2C). Western blotting showed that addition of b-FGF to PF treatment partially restored the $p$-ERK level, but not the $p$-AKT level, And adding AZD4547 to $\mathrm{PF} / \mathrm{b}$-FGF treatment suppressed p-ERK again (Figure 2D). 


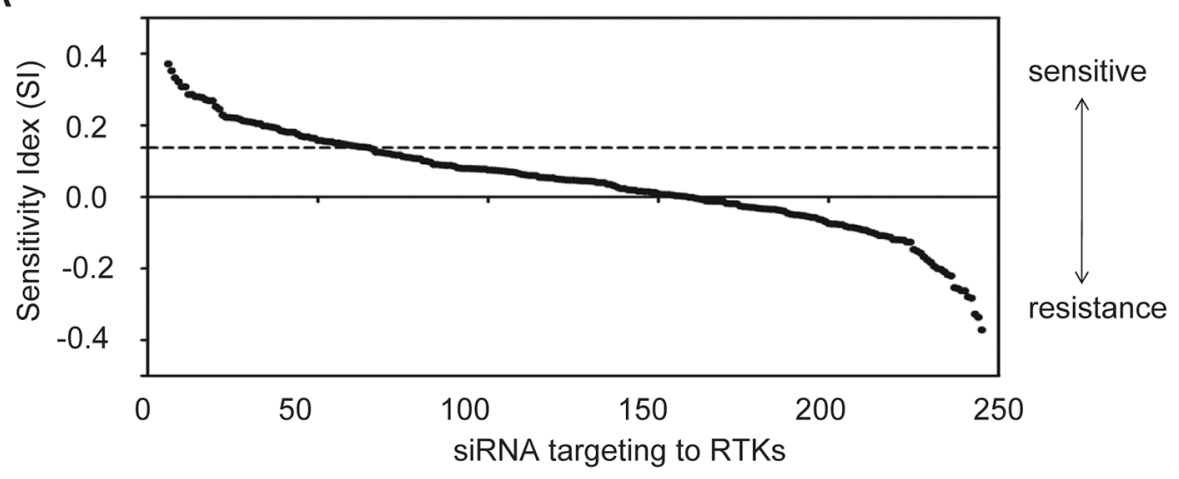

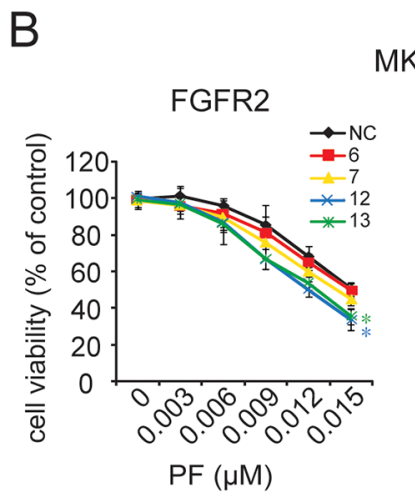

MKN-45

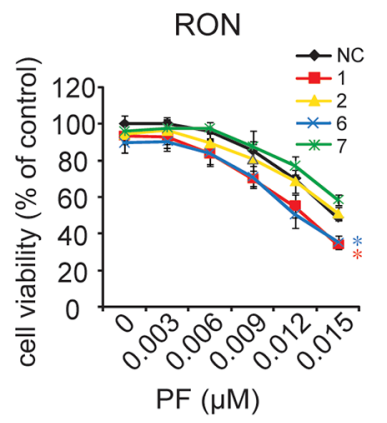

EPHA1
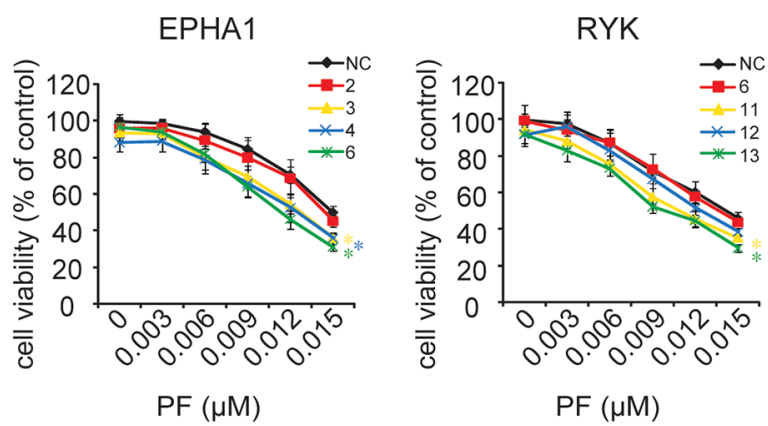

C

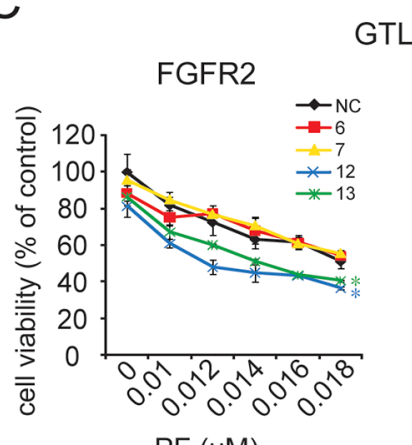

GTL-16
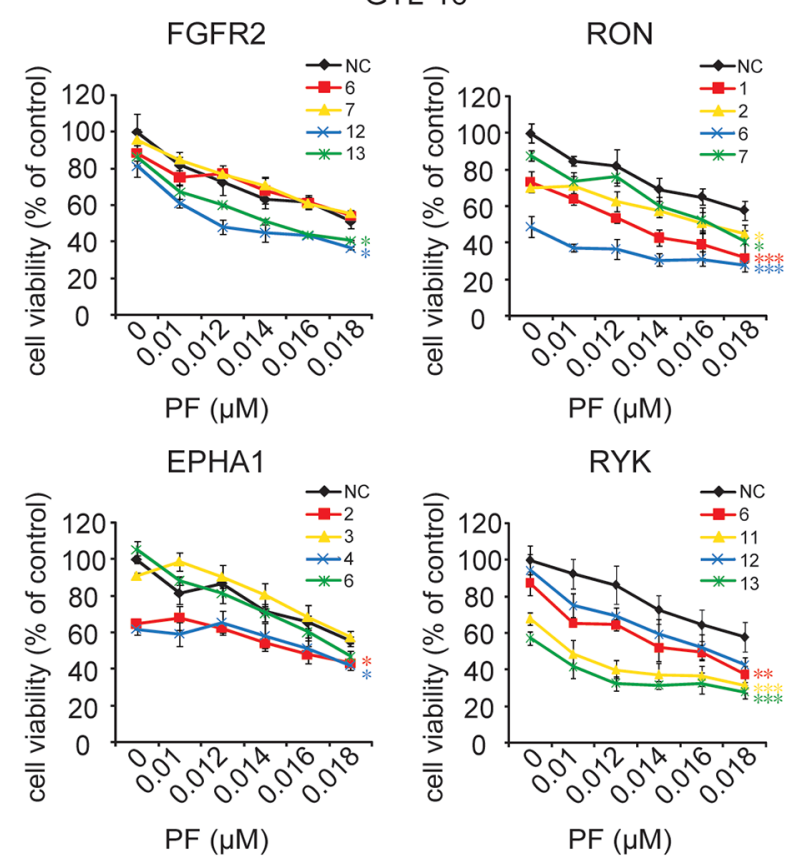

Figure 1: siRNA library screening identified gene candidates that could be targeted to sensitize GC cells to PF. A. The combined effect of siRNA and PF was assessed using the SI. Positive SI scores represented the sensitizing effect, and negative SI scores indicated antagonism to the treatment. In this study, SI scores in the upper quartile (above dashed line) were considered as conferring a strong drug-sensitizing phenotype. B. and C. Silencing of EPHA1, RYK, RON, or FGFR2 showed significant enhancement of PF effectiveness. MKN-45 and GTL-16 cells were transfected with non-targeting control (NC) siRNA and 4 siRNA species targeting each gene. Cell viability was measured using the CCK-8 cell-proliferation assay after 4 days of drug exposure. The percentage of viable cells is shown relative to untreated non-targeting controls. Values are the mean $\pm \mathrm{SD}$ of 3 replicates; ${ }^{*}, P<0.05,{ }^{* *}, P<0.01,{ }^{* * *}, P<0.001$, compared with the PF-treated NC group.

These findings indicated that activation of FGFR2 signaling pathway rescued MET+ GC cells from PF sensitivity, significantly.

\section{MSP fails to reactivate fl-RON in the presence of $\mathrm{PF}$}

RON and MET belong to the same subfamily. It is clear that RON actively crosstalks with MET, and takes part in the regulation of tumorigenic activity [18]. Our previous data showed that knockdown of RON sensitized GC cells to PF. Unexpectedly, treating with MSP, the ligand of fl-RON, resulted in no increase of PF-resistant MKN-45 cell clones (Figure 3A). Cell viability assay in GTL-16 cells showed that MSP did not affect PF-induced inhibition of cell growth, neither (Figure 3B). Then we detected the phosphorylation status of RON and downstream signaling molecules with the treatment of PF and MSP. The western blot assay revealed that $\mathrm{PF}$ at $0.04 \mu \mathrm{M}$ not merely blocked activation of MET, but also resulted in decreased fl-RON 


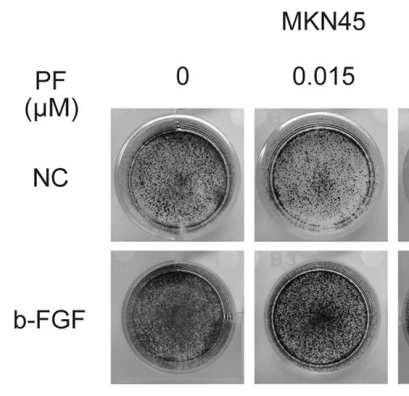

B

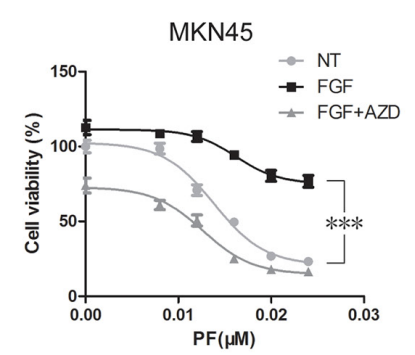

0.02
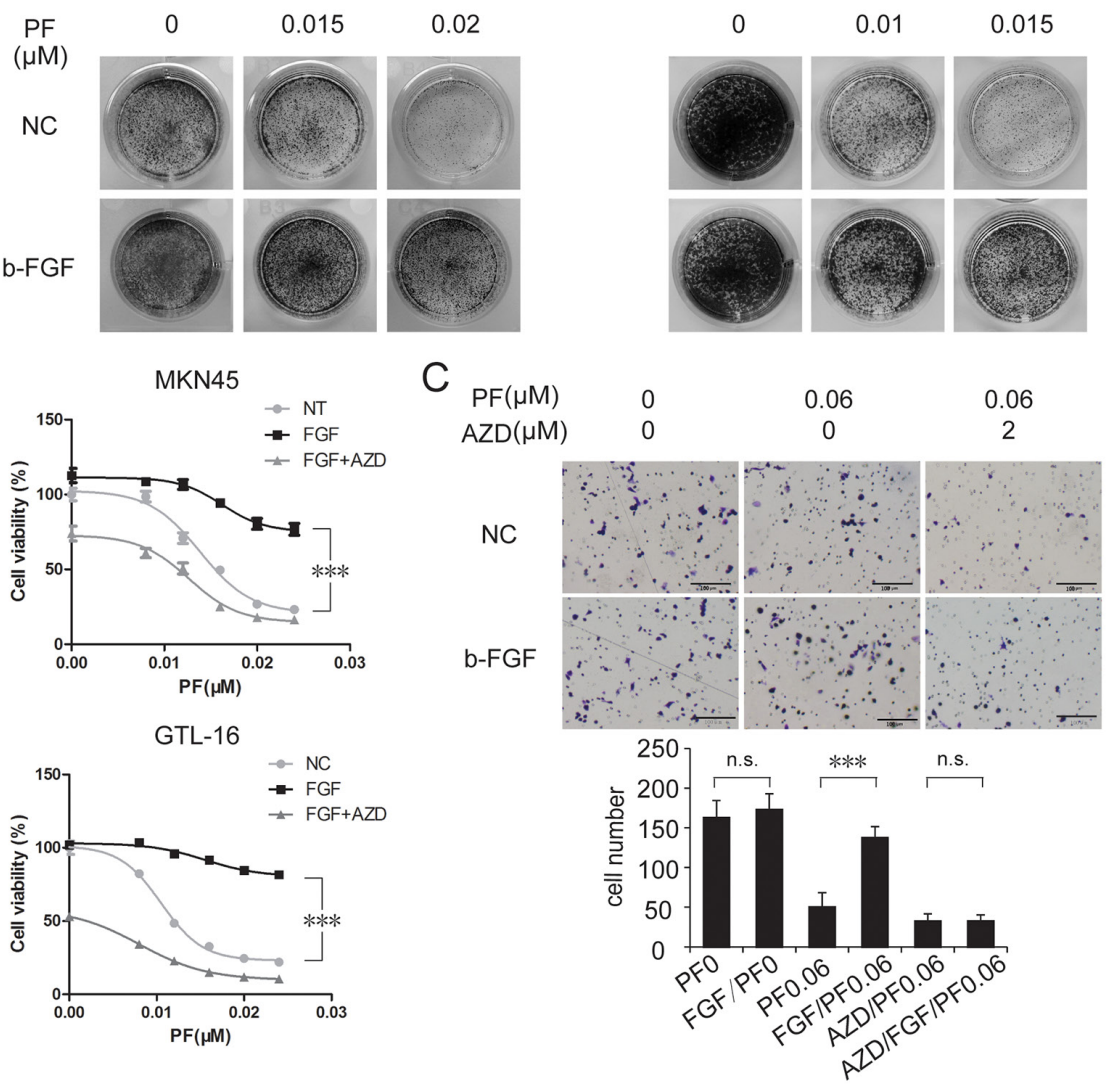

$\mathrm{D}$

MKN45

GTL-16

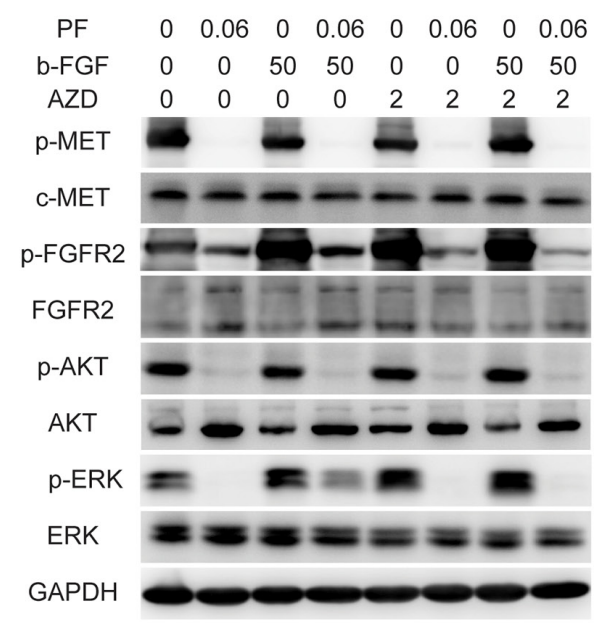

$\begin{array}{llllllll}0 & 0.06 & 0 & 0.06 & 0 & 0.06 & 0 & 0.06 \mu \mathrm{mol} / \mathrm{L}\end{array}$

$\begin{array}{lllllllll}0 & 0 & 50 & 50 & 0 & 0 & 50 & 50 & \mathrm{ng} / \mathrm{mL}\end{array}$

$\begin{array}{llllllllll}0 & 0 & 0 & 0 & 2 & 2 & 2 & 2 & \mu \mathrm{mol} / \mathrm{L}\end{array}$

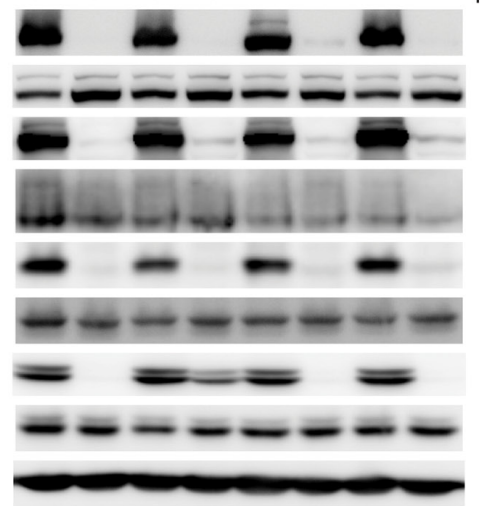

Figure 2: B-FGF-induced FGFR2 activation promoted PF unresponsiveness in MET-addicted GC cell lines. A. b-FGF induced PF resistance in MET-amplified cancer cell lines. Colony formation assays showed that stimulation of b-FGF resulted in obvious increase in PF-resistant cell clones. B. Combination of AZD4547 and PF significantly increased growth inhibition of MET-amplified cancer cells. MKN-45 and GTL-16 cells were exposed to PF $(0-0.024 \mu \mathrm{M})$ or a combination of PF and b-FGF $(50 \mathrm{ng} / \mathrm{mL})$ or a combination of $\mathrm{PF} / \mathrm{b}-\mathrm{FGF}$ and AZD4547 (5 $\mu \mathrm{M})$. Cell viability was measured using the CCK-8 cell-proliferation assay after 3 days of drug exposure. Values are the mean \pm SD of 3 replicates. C. Activation of FGFR2 signaling pathway attenuated PF-induced suppression of cell motility. Transwell migration assays of GTL-16 cells were performed with the treatment of PF $(0.01 \mu \mathrm{M})$, b-FGF 50 (ng/ml) and AZD4547 (2 $\mu \mathrm{M})$ for 48 hours. The results are representative images of migration assays, Bars, $50 \mu \mathrm{m}$ (top), and quantification of cells (bottom). Values are the mean \pm SD of 3 assays; $* * *, P<0.001$, n.s., no statistical significance. D. b-FGF conferred PF resistance by restoring ERK activation. Cells were pretreated with TKIs for 6 hours, and then stimulated with b-FGF for 30 minutes before whole cell lysates were collected. Western blot showed that ERK, but not AKT, reactivated after adding b-FGF to the treatment of PF. 


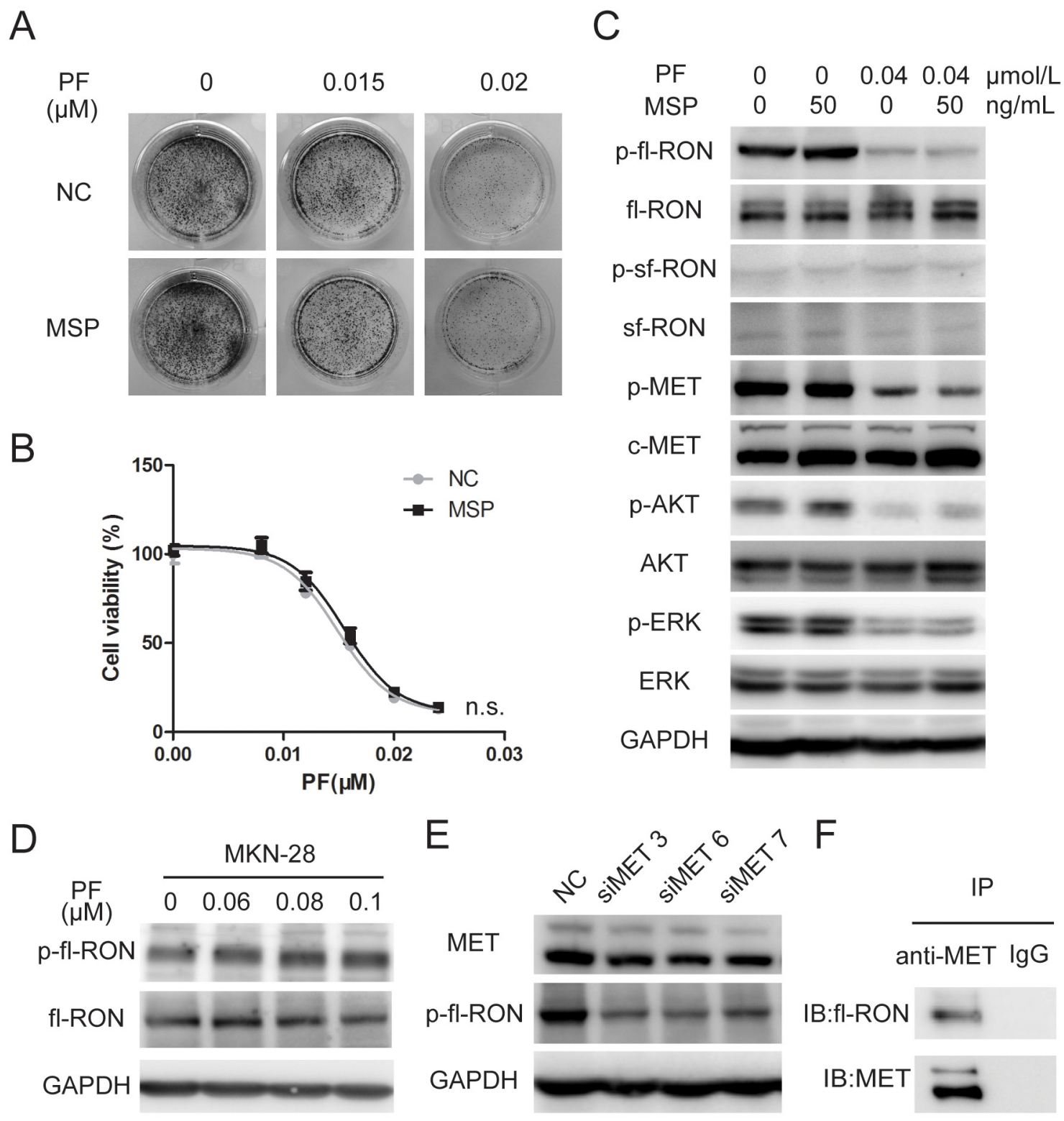

Figure 3: MSP did not contribute to PF unresponsiveness in MET-addicted GC cell lines. A. Colony formation assays showed that stimulation of b-FGF resulted in no increase in PF-resistant MKN-45cell clones. B. MSP hardly influence growth inhibition of MET-amplified cancer cells by PF. GTL-16 cells were exposed to PF (0-0.024 $\mu \mathrm{M})$ or a combination of PF and MSP (50 ng/mL). Cell viability was measured using the CCK-8 cell-proliferation assay after 3 days of drug exposure. Values are the mean \pm SD of 3 replicates. C. MSP failed to rescue $p$-fl-RON from PF exposure. MKN-45 cells were pretreated with PF $(0.04 \mu \mathrm{M})$ and MSP $(50 \mathrm{ng} / \mathrm{mL})$ before whole cell lysates were collected. Western blot analysis was conducted to examine the status of $p$-RON, $p$-MET, $p$-AKT, and $p$-ERK. D. PF did not inhibit phosphorylation of fl-RON in cell line with low expression of MET. MKN-28 cells were pretreated with PF $(0.06-0.1 \mu \mathrm{M})$, then Western blot analysis was conducted to examine the status of RON and $p$-RON. E. Knockdown of MET weaken phosphorylated fl-RON. MKN-45 cells were transfected with $20 \mathrm{nM} 3$ distinct siRNA species targeting MET or NC siRNA. MET and phosphorylated fl-RON was measured by Western blot 48 hours later. F. Co-immunoprecipitation indicated that MET and fl-RON formed heterodimers in MKN-45 cells. Irrelevant IgG serves as a negative control.

phosphorylation (Figure 3C). It is intriguing that $\mathrm{PF}$, regarded as a highly selective inhibitor to MET, could cause decreased fl-RON phosphorylation. We performed a series of experiment to investigate that whether PF targets fl-RON directly or PF cause decreased fl-RON phosphorylation indirectly through other mechanisms. As shown in Figure 3, PF $(0-0.1 \mu \mathrm{M})$ treatment in MKN-28 cells, a cell line with weak MET expression (Supplementary Figure S1), showed no inhibition to phosphorylation of fl-RON (Figure 3D). However, silencing MET via siRNA resulted in decreased 
fl-RON phosphorylation in MKN-45 cells (Figure 3E). This suggested the inhibition of PF to fl-RON may be depending on the status of MET. Co-immunoprecipitation analysis indicated that RON and MET could form heterodimers in GC cells, suggesting a transactivation of RON by MET (Figure 3F). MSP was unable to reactivate full-length RON and its downstream signaling when they were suppressed by PF (Figure 3C). Taken these data together, we concluded that in MET-addicted GC cells, fl-RON phosphorylation was inhibited by PF indirectly, probably through the inhibition of transactivation of RON and MET. And stimulation with MSP could not rescue the inhibition cause by PF.

\section{Upregulation of sf-RON impairs PF-induced inhibition of growth and migration in MET-addicted tumor cells}

MSP stimulation is not the only cause of RON pathway activation. Compared with fl-RON, sf-RON lacks most of the extracellular part. Thus, sf-RON proteins form constitutively active dimmers without the requirement for MSP [16]. Through Western blot assay, we noticed that phosphorylation of sf-RON was not influenced by $\mathrm{PF}$ (Figure 3C). To prove our conjecture that sf-RON contributes to PF resistance, the growth inhibitory effects of PF on sf-RON overexpressed cells and control cells were assessed through colony formation assay and cell proliferation inhibition assay. As shown in Figure 4A and Supplementary Figure S4A, in a panel of previously defined MET amplified cancer cell lines MKN-45, GTL-16 and EBC-1 [20, 28, 29], upregulation of sf-RON resulted in increased numbers of PF-resistant colonies. The inhibition rate was reduced significantly in sf-RON overexpressed groups compared with control groups $(P<0.001)$ (Figure $4 \mathrm{~B}$ and Supplementary Figures S4B). Western blot analysis demonstrated that, when exposed to PF, $p$-AKT and $p$ ERK were blocked completely in control group cells, while $p$-ERK was sustained at a certain level in sf-RON overexpressed GTL-16 cells, and both $p$-AKT and $p$-ERK was sustained at a certain level in sf-RON overexpressed EBC-1 cells (Figure 4F and Supplementary Figure S4F).

Similar to MET, RON is also implicated in tumor invasion and metastasis [30, 31]. Sf-RON expression increased motility of tumor cells [19]. Therefore, we assessed the potential effect of sf-RON on PF-induced motility suppression. In vitro transwell assay demonstrated that sf-RON overexpression remarkably enhanced the ability of GTL-16 cells to move through filter micropores. As expected, motility of these sf-RON overexpressed cells was not affected by PF at $0.06 \mu \mathrm{M}$, while migration of cells in the control group was significantly suppressed (Figure 4D). Similar to GTL-16 cells, sf-RON expressing in EBC-1 weakened PF induced suppression of cell motility (Supplementary Figure S4D).
To overcome PF resistance induced by sf-RON, we tested the efficacy of BMS777607, a small molecular inhibitor targets both RON and MET [32], on sf-RONoverexpressed GTL-16 and EBC-1 cells. Through proliferation inhibition assay, we found that the sensitivity of sf-RON cells to BMS777607 was consistent with that of NC cells (Figure 4C and Supplementary Figure S4C). Transwell assay revealed that the excessive motility triggered by sf-RON was significantly abrogated by BMS777607 (Figure 4E and Supplementary Figure S4E). Indeed, inhibiting sf-RON activation by BMS777607 significantly restored PF effectiveness on proliferation and migration in MET + cells, which were associated with their further blocking the downstream AKT and ERK signaling (Figure 4G and Supplementary Figure S4G). Taken together, these results implied that sf-RON pathway serves as a signaling compensatory mechanism, conferred resistance to MET inhibition.

\section{Upregulation of sf-RON contributes to $\mathbf{P F}$ resistance in vivo}

In order to confirm causal relationship between upregulation of sf-RON and PF resistance in vivo, firstly, xenograft tumors on immunocompromised mice were established with sf-RON overexpressed and NC GTL-16 cells. Mice were treated daily with vehicle (normal saline) or PF $(4 \mathrm{mg} / \mathrm{kg})$ by oral gavage 7 days after the implantation of tumor fragments. After treating for 24 days, the tumor volume percent $(\%)$ inhibition value reached $85.7 \%$ in the $\mathrm{NC}$ group and $70.2 \%$ in the sf-RON group, (Figure $5 \mathrm{~A}$ ). The average tumor weight was heavier in the sf-RON-PF group than in the NC-PF group (average tumor weight 0.25 \pm 0.05 g vs. $0.14 \pm 0.09 \mathrm{~g} ; n=6 ; P=0.039$; Figure $5 \mathrm{~B}$ ), while the tumor weights in the sf-RON-vehicle group and NC-vehicle group were comparable, with no statistical differences (average tumor weight $0.53 \pm 0.11 \mathrm{~g}$ vs. $0.54 \pm 0.12 \mathrm{~g} ; n=6 ; P=0.47$; Figure 5B). The extent of phosphorylated ERK and Ki67 in tumor xenografts was examined in formalin-fixed paraffin-embedded tumor sections (Figure 5C). P-ERK was totally suppressed by $\mathrm{PF}$ in the NC group, while it was present in samples from the sf-RON-PF group. There were fewer cells with Ki67 staining in the NC-PF group than in the sf-RON-PF group.

Secondly, an in vivo murine experimental metastasis assay was performed by using sf-RON transformed GTL16 cells and $\mathrm{NC}$ cells. In this assay, following injection of $1 \times 10^{6}$ cells into the caudal vein of nude mice, animals were treated daily with vehicle (normal saline) or PF ( $1 \mathrm{mg} / \mathrm{kg})$ by oral gavage for 45 days later. The results showed that the number of pulmonary metastatic nodules decreased significantly in the NC-PF group $(p<0.001)$, while the number of pulmonary metastatic nodules in sf-RON-PF group and sf-RON-vehicle group was similar ( $p=0.177$ ) (Figure 5D and 5E). Figure 5F 
A

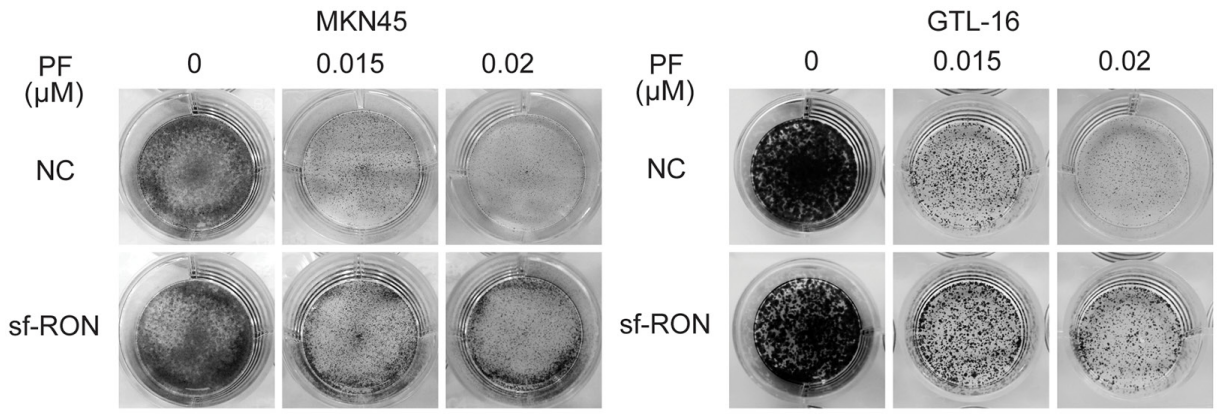

B

D

E

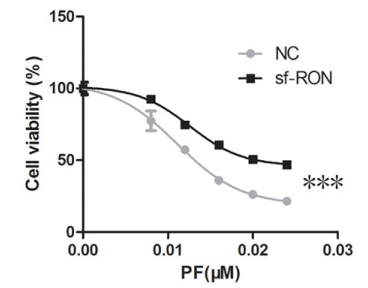

$\mathrm{PF}$
$(\mu \mathrm{M})$
$\mathrm{NC}$
sf-RON
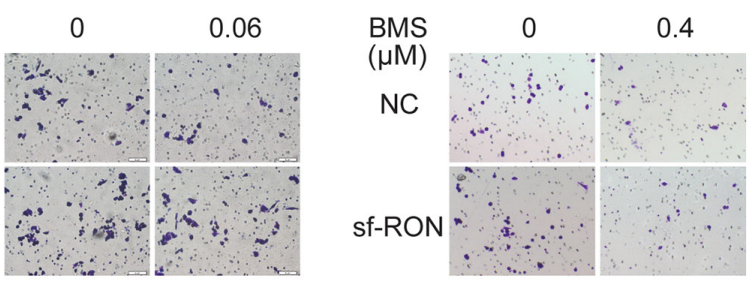

C
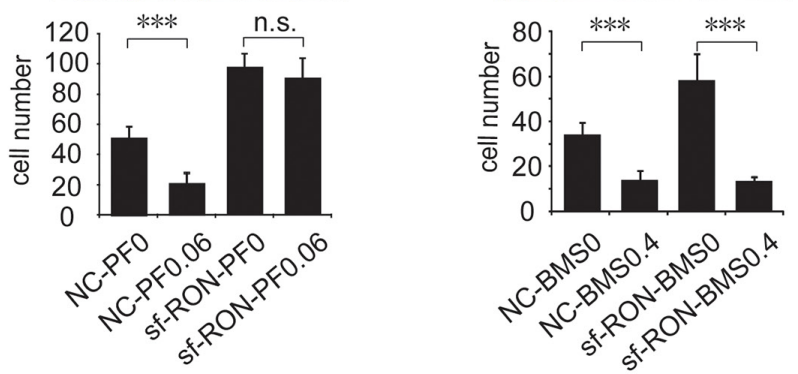

$\mathrm{F}$

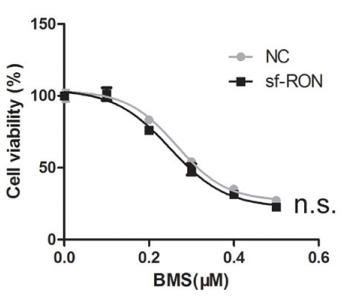

G

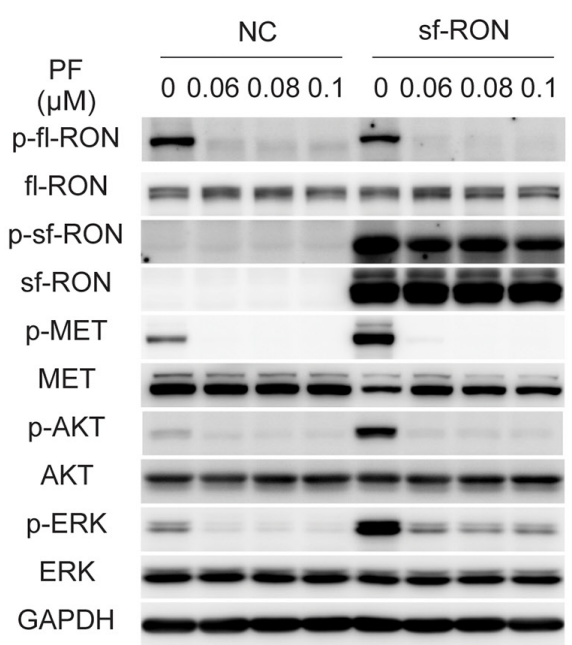

BMS $(\mu \mathrm{M})$
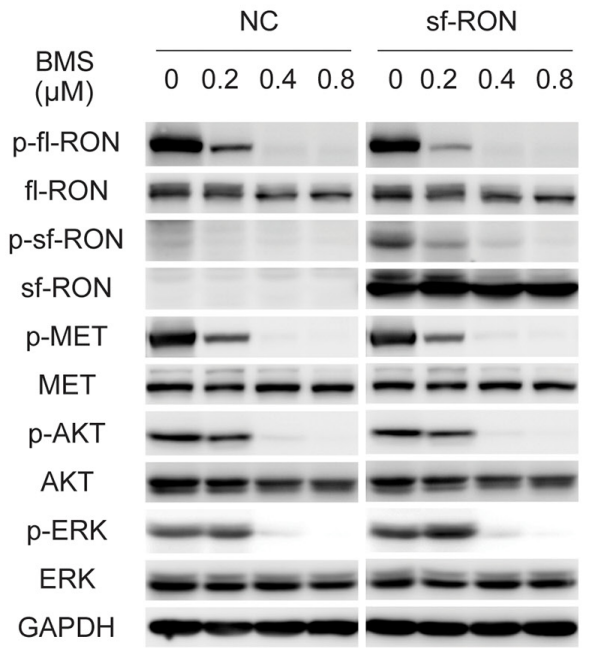

Figure 4: Upregulation of sf-RON attenuated PF-induced inhibition of cell proliferation and motility. A. and B. sf-RON induced PF resistance in MET-amplified GC cell lines. A, Colony formation assays showed that PF-resistant cell clones in the sf-RON group were greater than those in the NC group. B, cells were treated with increasing doses of PF $(0-0.024 \mu \mathrm{M})$, followed by CCK- 8 cellproliferation assay at 72 hours. C. MET/RON dual inhibition suppressed viability of cells with sf-RON overexpression effectively. Cells were treated with BMS $(0-0.5 \mu \mathrm{M})$, followed by CCK-8 cell-proliferation assay at 72 hours. D. and E. BMS, but not PF, abrogates sfRON-induced extra cell motility. Transwell migration assays of GTL-16 cells were performed with the treatment of $0.06 \mu \mathrm{M}$ PF (D) or 0.4 $\mu \mathrm{M}$ BMS (E) for 48 hours. Representative images of migration assays and statistics in bar graphs as indicated. Bars, $50 \mu \mathrm{m}$. Values are the mean \pm SD of 3 assays; ***, $P<0.001$, n.s., no statistical significance. $\mathbf{F}$. sf-RON confers PF resistance by restoring ERK activation. G. BMS total blocked phosphorylation of MET/RON and downstream signaling. Cells in the NC group and sf-RON group were pretreated with PF (F) or BMS (G) for 6 hours before whole cell lysates were collected. Western blot analysis was conducted to examine the status of $p$-RON, $p$-MET, $p$-AKT, and $p$-ERK. 
A

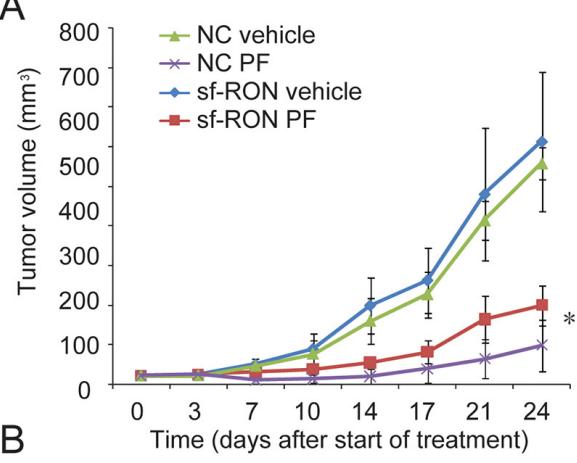

日

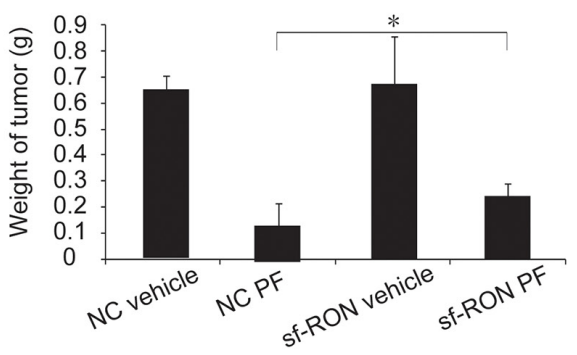

D

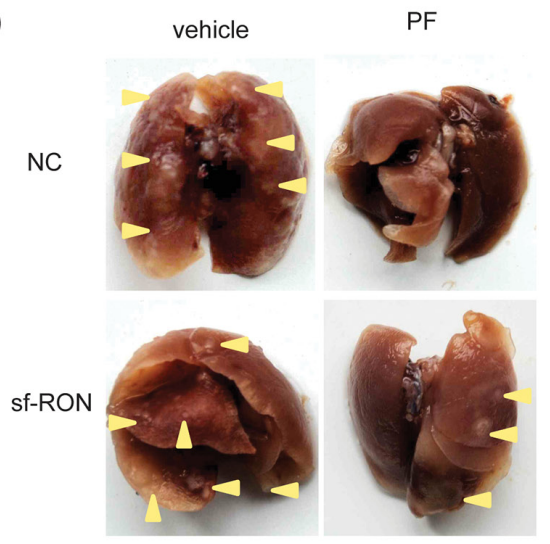

$E$

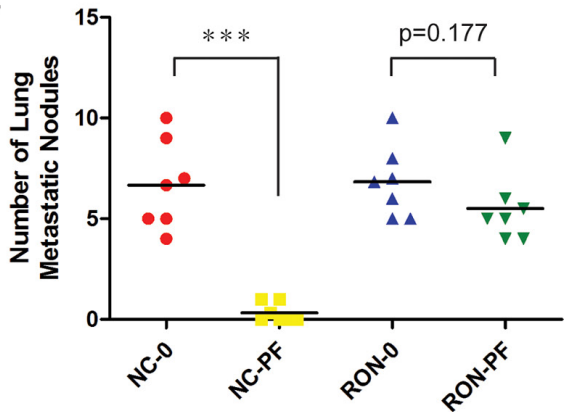

C
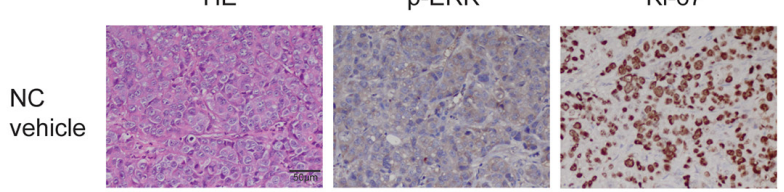

$\mathrm{NC}$
$\mathrm{PF}$

sf-RON
vehicle
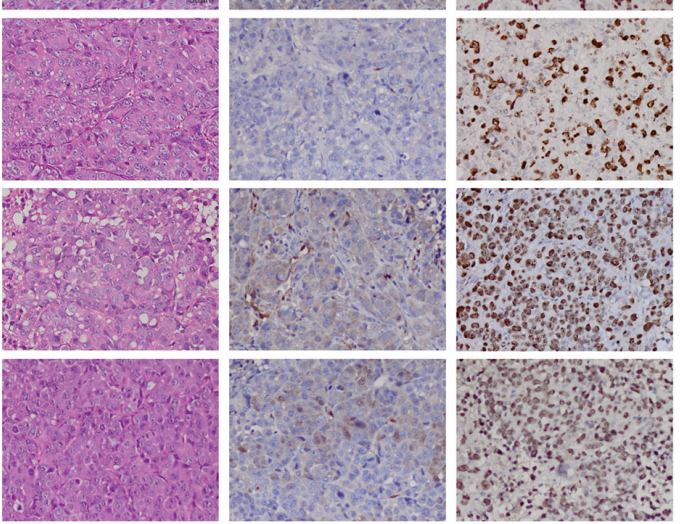

$\mathrm{PF}$
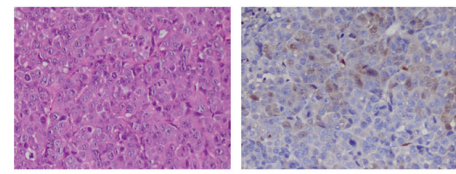

$\mathrm{F}$
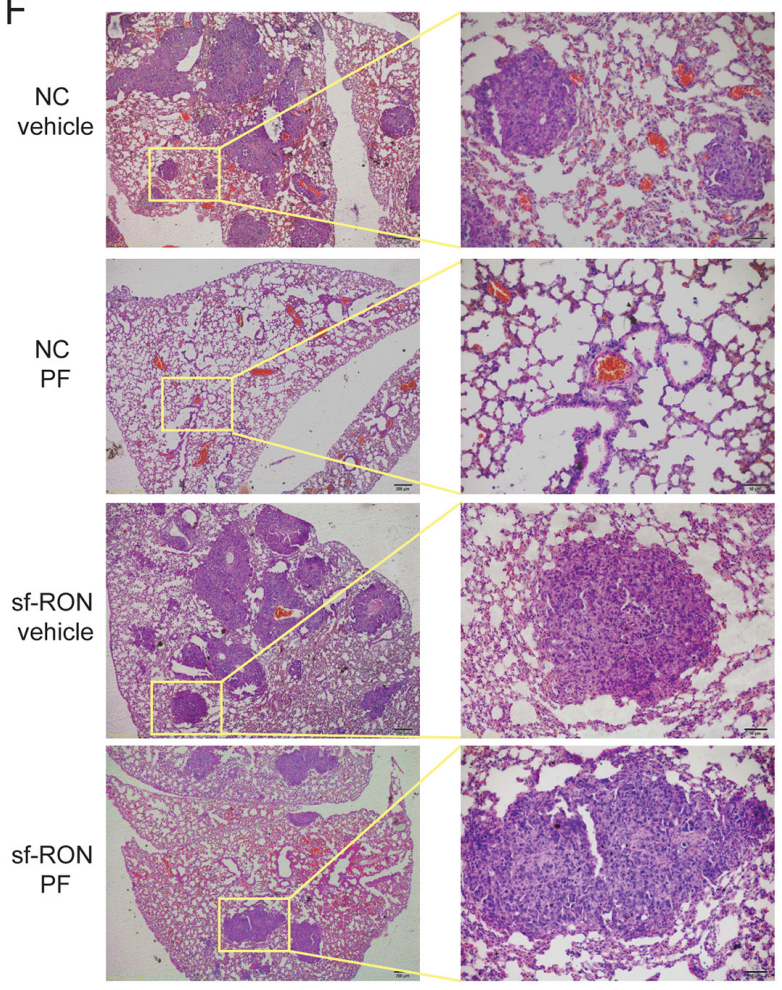

Figure 5: Upregulation of sf-RON contributed to PF resistance in vivo. A. Xenograft tumors on male nude mice were established with sf-RON-overexpressed GTL-16 cells and NC GTL-16 cells. Mice were treated for 7 days/week with either vehicle control (normal saline) or $4 \mathrm{mg} / \mathrm{kg}$ PF by oral gavage started at 7 days after implantation (day 0 ). Tumor size was measured twice per week. Values are mean $\pm \mathrm{SD}(n=6$ /group); *, $P<0.05, \mathrm{NC}$ PF group versus sf-RON PF group. B. 24 days after the start of treatment, the mice were euthanized. The weight of the tumors in each group was measured. Values are mean $\pm \mathrm{SD}(n=6 /$ group$) ; *, P<0.05, \mathrm{NC}$ PF group versus sf-RON PF group. C. Tumor tissue from xenografts treated for 24 days with the indicated drug regimens was evaluated by immunohistochemical staining for $p$-ERK and Ki67. Representative images as indicated; Original magnification, $\mathrm{x}$ 400, Scale bar, $50 \mu \mathrm{m}$. D, E, F. PF significantly reduced pulmonary metastatic nodules in NC PF group, but not in sf-RON PF group. D, Representative lungs from each group as indicated. $\mathrm{E}$, The number of pulmonary metastatic nodules in each group was calculated. Values are mean $\pm \mathrm{SD}(n=6 /$ group $) ; * * *, P<0.001, \mathrm{NC} \mathrm{PF}$ group versus NC vehicle group. F, Representative results of histological examination of mouse lungs for metastatic nodules in 4 groups. Left panel, Original magnification, x 100, Scale bar, $200 \mu \mathrm{m}$; right panel, Original magnification, x 400, Scale bar, $50 \mu \mathrm{m}$. 
showed representative images of the primary nodules of 4 groups. These results suggest that overexpression of sf-RON sustained cell proliferation and migration, which contributed to $\mathrm{PF}$ resistance in vivo.

\section{Sf-RON is frequently up-regulated in human GC samples}

The above results demonstrated that activation of sf-RON signaling pathways confer resistance to MET inhibitor. To further validate the clinical significance of these findings, we analyzed expression of both transcripts of RON and FGFR2 in GC tissue samples, especially in MET + samples. Firstly, the expression of MET, RON, and FGFR2 were assessed by immunohistochemistry (IHC) in 132 primary GC surgical samples. RON and FGFR2 were found to be highly expressed in 70.5\% (93/132) and 19.7\% (26/132) of specimens, respectively. There were more RONpositive samples in patients with MET+ tumors than in those with MET-negative tumors $(94.7 \%$ vs. $66.4 \% ; P=0.013$; Figure 6A and Table 1). While the positive expression rate of FGFR2 in MET+ cases was similar to that in METnegative cases $(26.3 \%$ vs. $30.8 \% ; P=0.532$; Table 1$)$.

Considering that sf-RON could not be distinguished by IHC, q-PCR was used to detect this isoform in GC cell lines and another 103 GC samples with specific primers. The status of MET expression in each sample was assessed through IHC (data not shown). Through statistical analysis, we found that the mRNA level of sf-RON was pervasively higher in GC cell lines compared with the immortality gastric cell line GES-1 (Supplementary Figure S5A). Moreover, the expression levels of the sf-RON in the tumor tissues significantly increased compared with those in adjacent non-tumor tissues, especially in MET+ samples ( $p=0.0017$ ) (Figure 6B and Supplementary Figure S5B). Then we compared the relative expression levels of sf-RON between the paired tumor and adjacent non-tumor tissues. Significant upregulation of sf-RON expression in paired GC/non-tumor samples was defined as a $\log _{2}$ fold change $>1$ (i.e., 2-fold), and downregulation was defined as a $\log _{2}$ fold change $<-1$. As shown in Supplementary Figure S5C, in the total $103 \mathrm{GC}$ samples, the proportion of specimens with sf-RON upregulation was $45.6 \%$, the proportion of specimens with sf-RON downregulation was $14.7 \%$, and the expression level of sf-RON was unchanged in the rest $41.7 \%$ ones. Furthermore, we found out that in MET+ GCs, the proportion of specimens with sf-RON upregulation (52.9\%) was much higher than that with sf-RON downregulation (5.9\%), while the proportion of specimens with sf-RON upregulation in MET- GCs was 39.2\% (Figure 6C).

\section{DISCUSSION}

Although MET kinase is regarded as one of the most promising therapeutic targets in gastric cancer, there are still a considerable number of MET + patients who do not respond to anti-MET agents. Elucidating the mechanisms of intrinsic resistance to anti-MET therapy will be of great value in implementation of more efficient drug combination strategies. Through a siRNA library screening that targeted most genes encoding RTKs and subsequent validation, we found that knockdown of 4 RTK receptors (FGFR2, RON, EPHA1, and RYK) via siRNA sensitized MET-addicted tumor cells to PF, while b-FGF-induced activation of FGFR2 conferred resistance to the PF. Consistent with our work, a previous study observed that MET-addicted cancer cells could be rescued by ligands of FGFR from crizotinib sensitivity [13]. However, treating with ligands of the other 3 receptors failed to cause PF resistance. We speculated that ligands stimulation may not induce these RTKs pathways activation, with the treatment of MET inhibitor.

Of the 3 novel finding hits, we focused on RON pathway. It have been proved that RON actively crosstalks with MET, and is essential to support the oncogenic phenotype of MET-addicted cancer cells [18, 33]. Our data confirmed that, in MET-amplified GC cells, fulllength RON was highly phosphorylated and could form heterodimers with MET. Phosphorylation of fl-RON was quenched by inhibition of MET via either specific TKI or siRNA. In the presence of MET inhibitor, MSP failed to reactivate fl-RON and downstream signaling. As a result, MSP was not able to rescue cancer cells from PF sensitivity. These findings imply that the activation of flRON strongly relies on the status of MET in MET-addicted cells. Thus, MSP induced activation of fl-RON pathway may be not involved in unresponsiveness to MET inhibitor.

Other than MSP induced dimerization and phosphorylation, aberrant activation of RON in cancer cells can also be achieved through overexpression of wild-type RON, and generation of activated mutations or isoforms [16]. Sf-RON, as a truncated transcript derived from the human $R O N$ gene, lacks the extracellular domains of this protein. Different from fl-RON, sf-RON proteins form disulfide-linked dimers automatically and constitutively phosphorylated, independently of MSP $[34,35]$. Sf-RON has been detected in several types of cancer, and drives tumor progression [19]. Here, we provide evidence that in MET-addicted GC cells, the level of phosphorylated sf-RON is barely influenced by MSP or PF. And for the first time, we observed that the expression levels of sf-RON in the GC tissues significantly increased compared with those in adjacent non-tumor tissues. Moreover, in more than $50 \%$ of MET+ GC samples the mRNA level of sf-RON was upregulated.

Because of constitutive kinase activity, sf-RON is thought to play an important role in aggressive behaviors [19]. Our data revealed that upregulation of sf-RON in MET-addicted tumor cells attenuated PF-induced suppression of cell proliferation and migration. Similar to MET, RON signalling is classically mediated by MEK/ ERK and PI3K/AKT pathways [16]. In the present study, we found that sf-RON maintain the activation of theses downstream signaling molecules, which could be inhibited 
A

Case 1
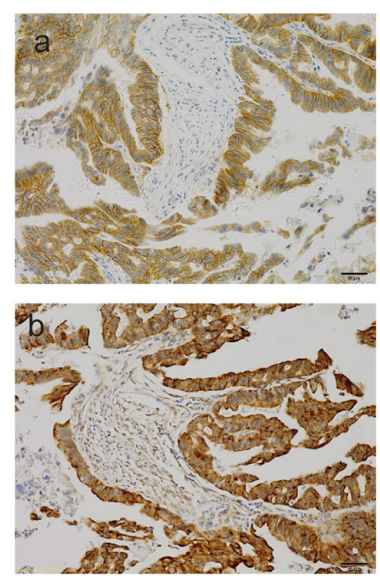

Case 2
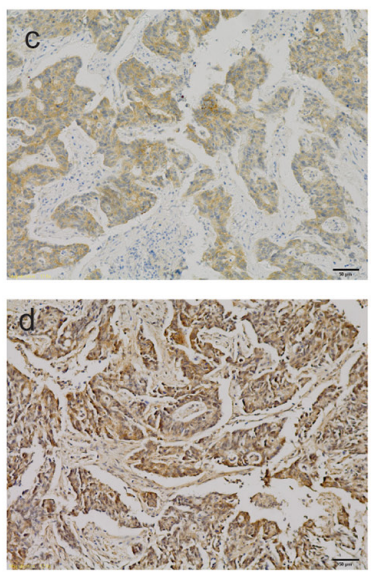

Case 3
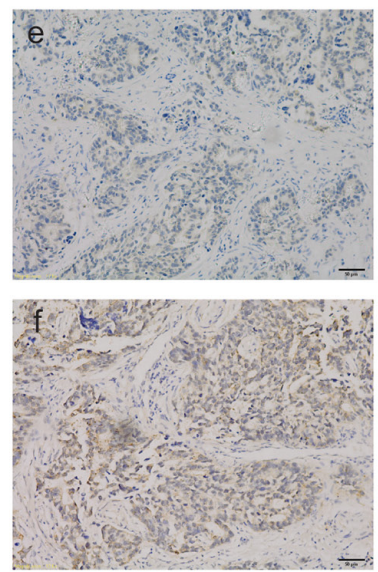

MET-
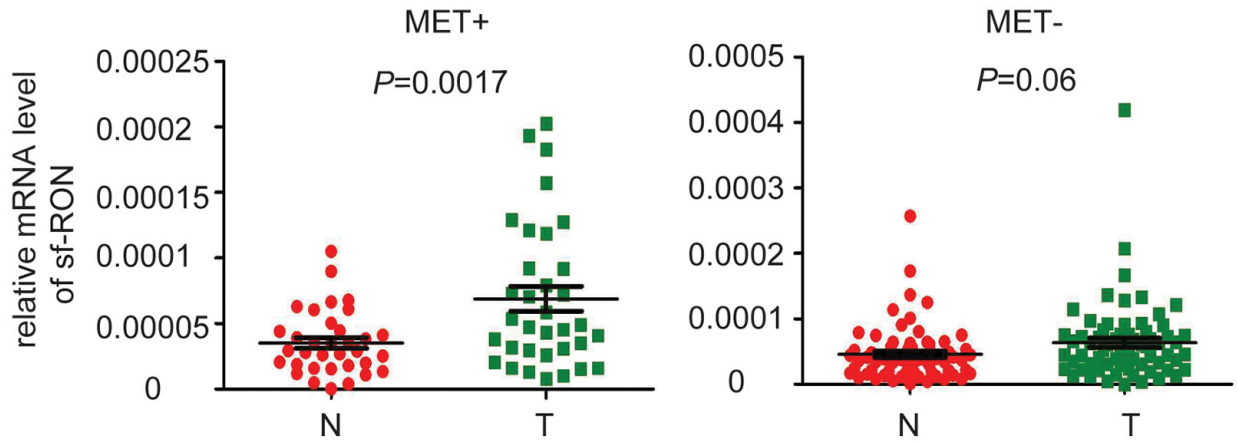

C

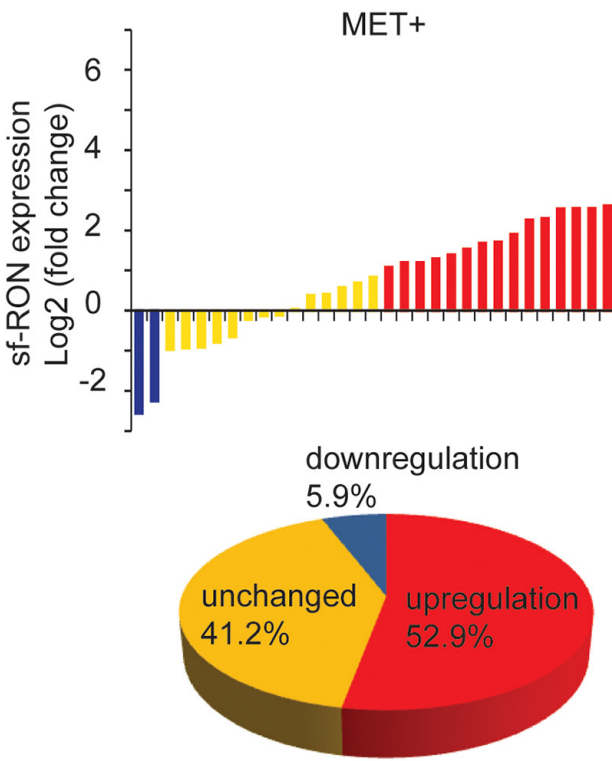

MET-
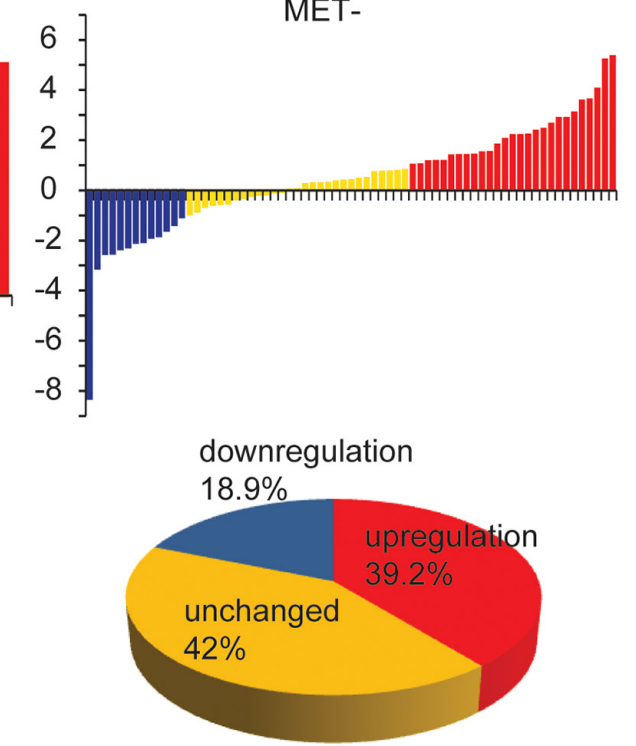

Figure 6: Expression of sf-RON was up-regulated in GC. A. co-expression of RON and MET in human GC. Representative images showed RON and MET co-expressed in human GC samples. (a) and (b) indicated strong positive expression of MET and RON in the same GC tissue; (c) and (d) indicated positive expression; (e) and (f) indicated negative expression. Original magnification, x 200. Scale bar, $50 \mu \mathrm{m}$. B. Upregulation of sf-RON correlated with MET status of GC. The expression levels of sf-RON in 103 paired GC and matched non-tumor tissues were determined by q-PCR. The relative mRNA level of sf-RON in GC was compared with that in paired non-tumor tissues. Statistical analysis of differences between the two groups was performed by paired Student's $t$-test and $P<0.05$ was considered statistically significant. C. sf-RON was up regulated in most MET-positive GCs. The data are expressed as the $\log _{2}$ fold change $(\Delta \mathrm{Ct}$ [GC/ Non]). Significant upregulation of sf-RON expression in paired tumor/non-tumor samples was defined as a $\log _{2}$ fold change $>1$. The pie chart displayed the proportions of GC samples showing upregulation (red), downregulation (blue), and unchanged (yellow). 
Table 1: Association of RON, FGFR2, and MET expression status

\begin{tabular}{|c|c|c|c|c|}
\hline \multirow[t]{2}{*}{ No. of Patients (\%) } & \multicolumn{3}{|c|}{ MET } & \multirow[t]{2}{*}{$P$ value } \\
\hline & Negative & Positive & Total & \\
\hline \multicolumn{5}{|l|}{ RON } \\
\hline Negative & $38(33.6)$ & $1(5.3)$ & $39(29.5)$ & 0.013 \\
\hline Positive & $75(66.4)$ & $18(94.7)$ & $93(70.5)$ & \\
\hline \multicolumn{5}{|l|}{ FGFR2 } \\
\hline Negative & $92(69.2)$ & $14(73.7)$ & $106(80.3)$ & 0.532 \\
\hline Positive & 21(18.6) & $5(26.3)$ & $26(19.7)$ & \\
\hline
\end{tabular}

Fisher's Exact test for all analyses.

via PF in control cells. These findings indicate that through generation of activated truncated isoform, RON pathway serves as a signaling compensatory mechanism, maintaining the growth and migration of PF-resistant cancer cells.

Rational combination strategy that simultaneously inhibits multiple targets or pathways may contribute to conquer drug resistance and improve efficacy of targeted therapeutics. Ichiro et al. have reported that dual inhibition of MET and RON signaling pathways by small-molecule inhibitor LY2801653 achieved dramatic antitumor effects in non-small cell lung cancer [36]. Here, we found that $\mathrm{PF}$ resistance induced by upregulation of sf-RON or activation of FGFR2 could be overcome by co-targeting MET with responsible bypass RTKs. Our observations might contribute to improve the efficacy of MET-targeted agents. It is worth noting that activation of sf-RON and FGFR2 pathways both reactive the downstream ERK signaling, which is originally suppressed by PF. MEK/ ERK pathway is also considered as crucial node in RAF kinase conferred resistance to MET inhibition [37, 38]. All of these observations emphasize the important role of MEK/ERK pathway in overcoming resistance to anti-MET therapy.

In conclusion, our findings indicate that sf-RON signaling is implicated in the unresponsiveness of MET+ gastric cancer to MET inhibitors. Considering the high proportion of sf-RON overexpresson in MET + gastric cancer samples, activation of this pathway might serve as a crucial signaling compensatory mechanism that attenuates the efficacy of anti-MET therapy. Further clinical studies are required to evaluate the efficacy of combination strategies co-targeting MET and RON signaling pathways on patients with GC.

\section{MATERIALS AND METHODS}

\section{Cell lines}

GC cell lines MKN-45 and MKN-28, and immortalized human gastric epithelial cell line GES-1, were obtained from 3D Biopharm Biotech Co. Ltd. (Shanghai,
China). NCI-N87, HGC-27, AGS, and SGC-7901 cell lines were obtained from the Cell Bank of Type Culture Collection of Chinese Academy of Sciences (Shanghai, China). SNU-216 and GTL-16 cell lines were gifts from the Medical College of Xiamen University (Xiamen, China) and AstraZeneca China R\&D Center (Shanghai, China). EBC-1 cell line was obtained from COBIOER BIOSCIENCES Co. Ltd (Nanjing, China). Cell lines were tested and authenticated by short tandem repeat DNA profiling analysis before execution of the experiments. Cells were cultured in Minimum Essential Medium (HGC27), F12K medium (AGS), Dulbecco's Modified Eagle's Medium (GTL-16), Eagle's Minimum Essential Medium (EBC-1) or Roswell Park Memorial Institute 1640 medium containing 10\% fetal bovine serum (Gibco, Carlsbad, CA, USA) and 1\% penicillin-streptomycin (Invitrogen, Carlsbad, CA, USA) at $37^{\circ} \mathrm{C}$ in a humidified atmosphere with $5 \%$ carbon dioxide.

\section{Drug preparations}

All tyrosine kinase inhibitors (TKIs) used were purchased from Selleck Chemicals (Houston, TX, USA). Recombinant human basic fibroblast growth factor (b-FGF) was purchased from PeproTech (Rocky Hill, NJ, USA). Recombinant human macrophage-stimulating protein (MSP), ephrinA1, and Wnt5a were from R\&D Systems (Minneapolis, MN, USA). Compounds were dissolved in 100\% dimethylsulfoxide (DMSO) (SigmaAldrich, St Louis, MO, USA) and diluted with culture medium to the desired concentration, with a final DMSO concentration $<0.2 \%(\mathrm{v} / \mathrm{v})$. DMSO was also added to control cells in culture.

\section{siRNA library screening and validation}

Screening was conducted using a siRNA library (QIAGEN, Hilden, Germany) containing 240 siRNAs with 4 unique siRNA species targeting each of 60 distinct RTKs. Scrambled control siRNA was used as the negative control (NC). On day 1, siRNA (final concentration = $5 \mathrm{nM}$ ) was first added to 96-well plates and incubated 
with HiPerFect Transfection Reagent (QIAGEN, Hilden, Germany) to allow formation of transfection complexes, then MKN-45 cells were seeded at 2500 per well. On day 2, cells were treated with PF04217903 (PF) $0.012 \mu \mathrm{M}$ or $0.2 \%$ DMSO alone (vehicle). Cell viability was assessed 4 days later using Cell Counting Kit-8 (CCK-8) Cell Viability Assay following the manufacturer's protocol. The assay was performed in triplicate. To evaluate gene targets that increase PF sensitivity or resistance, the sensitivity index (SI) was calculated as described [21, 39]. In brief, the effect of siRNA compared to negative siRNA control was designated $\mathrm{Rc} / \mathrm{Cc}$, and effect of the drugs on controltransfected cells was designated $\mathrm{Cd} / \mathrm{Cc}$. The expected combined effect of siRNA and drug on cell viability could be calculated by $\mathrm{Rc} / \mathrm{Cc} \times \mathrm{Cd} / \mathrm{Cc}$. The Observed combined effects of drug and siRNA on cell viability compared to negative siRNA control was designated $\mathrm{Rd} / \mathrm{Cc}$. Then, an index of antagonism or sensitivity (SI) for each siRNA was calculated by the Observed combined effect minus the Expected combined effect: $\mathrm{SI}=(\mathrm{Rc} / \mathrm{Cc} * \mathrm{Cd} / \mathrm{Cc})-(\mathrm{Rd} /$ $\mathrm{Cc})$. A positive SI score indicates a sensitizing effect and a negative SI score indicates antagonism to the treatment, an additional criteria that $\mathrm{Rd} / \mathrm{Cd}<0.95$ (for sensitizing siRNAs) was employed for hit selection. SI scores was ranked and those in the upper quartile were considered as conferring a strong drug-sensitizing phenotype. Another 4 distinct siRNA species (QIAGEN) targeting each gene were used to validate hits from the initial screen. The transfection process was consistent with that in the screening. Twenty four hours after transfection, cells were treated with vehicle or a dose range of $\mathrm{PF}(0.008-0.024 \mu \mathrm{M})$. Cell viability was assessed 4 days later through CCK-8 Assay.

\section{Cell viability and colony formation assay}

Cell line sensitivity to the indicated treatment was determined through Cell Viability Assay. In brief, cells were seeded at 2,500 cells per well in 96-well plates and incubated overnight. Cells were then treated with increasing concentrations of the indicated drugs and other agents for 72 hours. Treatments at each concentration were carried out in 6 replicate wells and repeated 3 times. Cell viability was determined using the CCK-8 according to the manufacturer's instructions. Colony formation assay was conducted as previously described [39]. Briefly, MKN-45 cells were seeded at $1 \times 10^{4}$ cells per well in triplicate in 12-well plates and cultured in the absence or presence of the drug and ligands for 10 days. After fixing the cells with $4 \%$ paraformaldehyde, cell clones were stained with $0.1 \%$ crystal violet.

\section{In vitro migration assays}

Cell migration was analyzed by using a BD Falcon Cell Culture Insert System (BD Biosciences, San Jose, CA, USA) with $8-\mu \mathrm{m}$ pores. For motility assays, $6 \times 10^{4}$ GTL-16 cells in $300 \mu \mathrm{L}$ of serum-free medium were seeded into upper inserts, with $600 \mu \mathrm{L}$ of $10 \%$ serum medium to the lower chamber. After treating with indicated drugs and agents for 48 hours, cells were fixed with $0.5 \mathrm{~mL}$ of $4 \%$ paraformaldehyde. Then, each well was washed 3 times with $1 \times$ PBS, and stained with $0.6 \mathrm{~mL}$ of $0.1 \%$ crystal violet solution. After removing the cells on the upper chamber using a cotton swab, the number of cells was counted at 5 fields per membrane at 200x magnification from each group of 3 independent experiments.

\section{Lentivirus production and transduction}

Virus packaging was performed in human embryonic kidney (HEK) $293 \mathrm{~T}$ cells after cotransfection of pWPXL-sf-RON or empty vector with the packaging plasmid psPAX2 (Addgene plasmid 12260; Didier Trono Lab, Cambridge, MA, USA) and the envelope plasmid pMD2.G (Addgene plasmid 12259, Didier Trono Lab) using Lipofectamine ${ }^{\circledR} 2000$ (Invitrogen, Carlsbad, CA, USA). Viruses were harvested 48 hours after transfection. Target cells, including MKN-45 and GTL-16 were infected with filtered lentivirus in the presence of $6 \mathrm{mg} / \mathrm{mL}$ Polybrene $^{\circledR}$ (Sigma-Aldrich, St Louis, MO, USA).

\section{Western blot and antibodies}

Cells were lysed in radioimmunoprecipitation assay buffer (Biyotime, Shanghai, China) supplemented with complete protease inhibitor cocktail (Roche, Basel, Switzerland). Protein concentrations were determined using the BCA protein assay kit (Biyotime, Shanghai, China). Western blot was carried out with $30 \mu \mathrm{g}$ total proteins and antibodies against MET, $p$-MET, $p$-FGFR2, AKT, $p$-AKT, ERK, $p$-ERK kinase, $\beta$-actin and GAPDH (Cell Signaling Technology, Cambridge, MA, USA), RON and $p$-RON (Santa Cruz Biotechnology, CA, USA), and FGFR2 (R\&D Systems, Minneapolis, MN, USA). Blots were probed with the indicated primary antibodies, then incubated with the horseradish peroxidaseconjugated secondary antibody and detected by enhanced chemiluminescence reagent (Pierce, Rockford, IL, USA).

\section{Immunoprecipitation}

The experiment was performed by using Pierce ${ }^{\mathrm{TM}}$ Classic Magnetic IP/Co-IP Kit (Pierce, Rockford, IL, USA), according to the manufacturer's instructions. In brief, cells were lysed in buffer ( $\mathrm{pH} 7.4)$ containing $0.025 \mathrm{M}$ Tris, $0.15 \mathrm{M} \mathrm{NaCl}, 0.001 \mathrm{M}$ EDTA, $1 \%$ NP40, $5 \%$ glycerol, protease and phosphatase inhibitor cocktails. 500 $\mu \mathrm{g}$ total protein were immunoprecipitated overnight at $4^{\circ} \mathrm{C}$ with the antibody against MET (Cell Signaling Technology, Cambridge, MA, USA). Add the antigen sample/antibody mixture (Section B) to the tube containing pre-washed magnetic beads and incubate at room temperature for 1 hour with mixing. The immunocomplexes were eluted 
by Elution Buffer, and immunoblotted to detect RON. Irrelevant IgG served as a negative control.

\section{Xenograft experiments}

Male athymic nude mice, aged 6 weeks, were purchased and raised under specific pathogen-free conditions at Shanghai Slac Laboratory Animal Co. Ltd. (Shanghai, China). All animal work must have been conducted according to relevant national and international guidelines. Firstly, Stable GTL-16 cell lines transfected with empty vector (GTL-16 NC) or sf-RON (GTL-16 sf-RON) were subcutaneously implanted into the right flanks region of the mice $\left(10^{7}\right.$ cells in $0.1 \mathrm{~mL}$ PBS), respectively. Once the volume of the tumor xenograft reached approximately $300-500 \mathrm{~mm}^{3}$, tumors were further established in the nude mice ( $n=6 /$ group) by subcutaneous implantation of tumor fragments $(\sim 2 \times 2 \times 2 \mathrm{~mm})$ obtained from donor mice. Treatment started 7days after implantation (day 0) with either PF (4 mg/kg, daily) or vehicle (normal saline, daily) by oral gavage for 24 days. Tumor volumes were determined from caliper measurements of tumor length (L) and width (W) according to the formula $\mathrm{LW}^{2} / 2$. Both tumor size and body weight were measured twice per week. Percent inhibition values were calculated as: $100 \times\left(1-\left(\left(\mathrm{PF}_{\text {final day }}\right.\right.\right.$ $\left.-\mathrm{PF}_{\text {day } 0}\right) /\left(\right.$ vehicle $_{\text {final day }}-$ vehicle $\left.\left.\left._{\text {day } 0}\right)\right)\right)$. Tumor volumes were analyzed using one-way analysis of variance (ANOVA). At the end of the treatment period, mice were humanely euthanized and the tumors were weighed and fixed in $10 \%$ buffered formalin for immunostaining. Tumor weights were compared using $t$ test.

\section{In vivo metastasis assays}

For in vivo murine Experimental metastasis assays, cells $\left(1 \times 10^{6}\right.$ per mouse $)$ were injected into the tail veins of nude mice, followed by treating animals with either PF $(1 \mathrm{mg} / \mathrm{kg}$, daily) or vehicle (normal saline, daily) by oral gavage. Six weeks later, all of the mice were sacrificed and the lungs were dissected and processed for standard histological studies. For histological analysis, the mouse organs were fixed in $10 \%$ formalin and paraffin-embedded and 4 serial sections were obtained from each sample. The sections were stained with hematoxylin and eosin (H\&E) and analyzed for the presence of metastases. The number of pulmonary metastatic nodules between PF-treating group and vehicle-treating group was compared using $t$ test.

\section{Patients and specimens}

All primary gastric adenocarcinomas specimens were obtained by surgical resection between 2007 and 2010 at Shanghai Cancer Center, Fudan University, Shanghai, China. Samples were acquired after informed consent was given, under the protocol approved by the Shanghai Cancer
Center research ethics committee. Tissue microarrays were constructed in collaboration with Shanghai Biochip (Shanghai, China), as described [39]. Additionally, a set of 103 fresh frozen GC samples was analyzed for expression of sf-RON and full-length RON (fl-RON).

\section{Immunohistochemistry and scoring system}

Paraffin embedded GC tissue sections were subjected to immunohistochemical assays using a MaxVision ${ }^{\mathrm{TM}}$ HRP-Polymer Detection System (Maixin, Fuzhou, China), as described previously [39]. Primary antibodies against MET (Roche, Basel, Switzerland), RON (Santa Cruz Biotechnology, Santa Cruz, CA, USA), and FGFR2 (R\&D Systems, Minneapolis, MN, USA) were used to assess the expression of MET, RON, and FGFR2 in 132 primary GC surgical samples. Primary antibodies against $p$-ERK (Cell Signaling Technology) and Ki67 (Dako, Denmark) were used on GC xenograft sections.

Slides were independently evaluated by 2 investigators who were blinded to the clinical information. The intensity of MET, RON, and FGFR2 membrane immunostaining and prevalence of these intensities in tumor cells were evaluated. Staining intensity ( 0 none, $1+$ weak, $2+$ moderate, $3+$ strong) and percentage of cells staining were independently scored. Samples that scored at least $2+$ in at least $50 \%$ of tumor cells were regarded as positive for each marker [40].

\section{RNA extraction and reverse transcription- polymerase chain reaction}

Total RNA was extracted from tumor tissues using Trizol Reagent (Invitrogen, Carlsbad, CA, USA), and reversely transcribed through PrimeScript ${ }^{\mathrm{TM}}$ reverse transcription-polymerase chain reaction (RT-PCR) kit (TaKaRa, Shiga, Japan) according to the manufacturer's instructions, as described [41]. mRNA expression levels were quantified with SYBR Premix Ex Taq kit (TaKaRa, Japan) on a 7900 Real-Time PCR System with SDS 2.3 software (Applied Biosystems) at the recommended thermal cycling settings: one initial cycle at $95^{\circ} \mathrm{C}$ for $10 \mathrm{~s}$ followed by 40 cycles of $5 \mathrm{~s}$ at $95^{\circ} \mathrm{C}$ and $31 \mathrm{~s}$ at $60^{\circ} \mathrm{C}$. To examine sf-RON transcript expression, specific primers were designed as follows: sense: $5^{\prime}-$

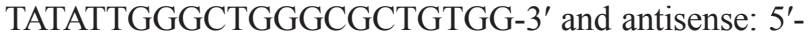
TACAATGGGGCACCATCCTG-3'. The expression level of sf-RON were normalised to the internal reference gene 18s rRNA (sense, 5' -GTAACCCGTTGAACCCCATT-3' ; antisense, 5' -CCATCCAATCGGTAGTAGCG-3') [42].

\section{Statistical analysis}

Statistical analyses were performed with the Statistical Package for the Social Sciences version16.0 (SPSS Inc., Chicago, IL, USA) or with GraphPad Prism 
version 5.0 (GraphPad Software, La Jolla, CA, USA). Quantitative variables were analyzed by Student's $t$ test or one-way ANOVA with Bonferroni post-test. Fisher's exact test was used to compare qualitative variables. Differences between cell viability and growth curves were analyzed by two-way ANOVA followed by Bonferroni's multiple comparison test. Two-tailed $P<0.05$ was considered statistically significant.

\section{ACKNOWLEDGMENTS AND FUNDING}

We are grateful to Prof. Xianghuo He (Shanghai Cancer Center, Fudan University, Shanghai, China), and Prof. Zhigang Zhang (Shanghai Cancer Institute, Ren Ji Hospital, Shanghai Jiao Tong University, Shanghai, China) for critically reading the manuscript. We thank Doctor Zebing Liu, and Doctor Jun Li for assistance in IHC assessment. We thank Yuhu Xin for technical assistance with Lentivirus production.

\section{CONFLICTS OF INTEREST}

The author(s) indicated no potential conflicts of interest.

\section{GRANT SUPPORT}

This work was supported by the National Science and Technology Major Projects of China (Grant No.: 2012ZX09303-018-002).

\section{REFERENCES}

1. Gherardi E, Birchmeier W, Birchmeier C, Vande Woude G. Targeting MET in cancer: rationale and progress. Nat Rev Cancer. 2012; 12:89-103.

2. Lee HE, Kim MA, Lee HS, Jung EJ, Yang HK, Lee BL, Bang YJ, Kim WH. MET in gastric carcinomas: comparison between protein expression and gene copy number and impact on clinical outcome. Br J Cancer. 2012; 107:325-333.

3. Deng N, Goh LK, Wang H, Das K, Tao J, Tan IB, Zhang S, Lee M, Wu J, Lim KH, Lei Z, Goh G, Lim QY, Tan AL, Sin Poh DY, Riahi S, et al. A comprehensive survey of genomic alterations in gastric cancer reveals systematic patterns of molecular exclusivity and co-occurrence among distinct therapeutic targets. Gut. 2012; 61:673-684.

4. Nakajima M, Sawada H, Yamada Y, Watanabe A, Tatsumi M, Yamashita J, Matsuda M, Sakaguchi T, Hirao T, Nakano H. The prognostic significance of amplification and overexpression of c-met and c-erb B-2 in human gastric carcinomas. Cancer. 1999; 85:1894-1902.
5. Lennerz JK, Kwak EL, Ackerman A, Michael M, Fox SB, Bergethon K, Lauwers GY, Christensen JG, Wilner KD, Haber DA, Salgia R, Bang YJ, Clark JW, Solomon BJ, Iafrate AJ. MET amplification identifies a small and aggressive subgroup of esophagogastric adenocarcinoma with evidence of responsiveness to crizotinib. J Clin Oncol. 2011; 29:4803-4810.

6. Kawakami H, Okamoto I, Arao T, Okamoto W, Matsumoto K, Taniguchi H, Kuwata K, Yamaguchi H, Nishio K, Nakagawa K, Yamada Y. MET amplification as a potential therapeutic target in gastric cancer. Oncotarget. 2013; 4:9-17.

7. Huang TJ, Wang JY, Lin SR, Lian ST, Hsieh JS. Overexpression of the c-met protooncogene in human gastric carcinoma-correlation to clinical features. Acta Oncol. 2001; 40:638-643.

8. Graziano F, Galluccio N, Lorenzini P, Ruzzo A, Canestrari E, D'Emidio S, Catalano V, Sisti V, Ligorio C, Andreoni F, Rulli E, Di Oto E, Fiorentini G, Zingaretti C, De Nictolis M, Cappuzzo F, et al. Genetic activation of the MET pathway and prognosis of patients with highrisk, radically resected gastric cancer. J Clin Oncol. 2011; 29:4789-4795.

9. Peters S, Adjei AA. MET: a promising anticancer therapeutic target. Nat Rev Clin Oncol. 2012; 9:314-326.

10. Iveson $\mathrm{T}$, Donehower RC, Davidenko I, Tjulandin $\mathrm{S}$, Deptala A, Harrison M, Nirni S, Lakshmaiah K, Thomas A, Jiang Y, Zhu M, Tang R, Anderson A, Dubey S, Oliner KS, Loh E. Rilotumumab in combination with epirubicin, cisplatin, and capecitabine as first-line treatment for gastric or oesophagogastric junction adenocarcinoma: an open-label, dose de-escalation phase $1 \mathrm{~b}$ study and a double-blind, randomised phase 2 study. Lancet Oncol. 2014; 15:1007-1018.

11. Shah MA, Wainberg ZA, Catenacci DV, Hochster HS, Ford J, Kunz P, Lee FC, Kallender H, Cecchi F, Rabe DC, Keer H, Martin AM, Liu Y, Gagnon R, Bonate P, Liu L, et al. Phase II study evaluating 2 dosing schedules of oral foretinib (GSK1363089), cMET/VEGFR2 inhibitor, in patients with metastatic gastric cancer. PLoS One. 2013; 8:e54014.

12. Kang YK, Muro K, Ryu MH, Yasui $H$, Nishina $T$, Ryoo BY, Kamiya Y, Akinaga S, Boku N. A phase II trial of a selective c-Met inhibitor tivantinib (ARQ 197) monotherapy as a second- or third-line therapy in the patients with metastatic gastric cancer. Invest New Drugs. 2014; 32:355-361.

13. Wilson TR, Fridlyand J, Yan Y, Penuel E, Burton L, Chan E, Peng J, Lin E, Wang Y, Sosman J, Ribas A, Li J, Moffat J, Sutherlin DP, Koeppen H, Merchant M, et al. Widespread potential for growth-factor-driven resistance to anticancer kinase inhibitors. Nature. 2012; 487:505-509. 
14. Holohan C, Van Schaeybroeck S, Longley DB, Johnston PG. Cancer drug resistance: an evolving paradigm. Nat Rev Cancer. 2013; 13:714-726.

15. Niederst MJ, Engelman JA. Bypass mechanisms of resistance to receptor tyrosine kinase inhibition in lung cancer. Sci Signal. 2013; 6:re6.

16. Yao HP, Zhou YQ, Zhang R, Wang MH. MSP-RON signalling in cancer: pathogenesis and therapeutic potential. Nat Rev Cancer. 2013; 13:466-481.

17. Catenacci DV, Cervantes G, Yala S, Nelson EA, El-Hashani E, Kanteti R, El Dinali M, Hasina R, Bragelmann J, Seiwert T, Sanicola M, Henderson L, Grushko TA, Olopade O, Karrison T, Bang YJ, et al. RON (MST1R) is a novel prognostic marker and therapeutic target for gastroesophageal adenocarcinoma. Cancer Biol Ther. 2011; 12:9-46.

18. Follenzi A, Bakovic S, Gual P, Stella MC, Longati P, Comoglio PM. Cross-talk between the proto-oncogenes Met and Ron. Oncogene. 2000; 19:3041-3049.

19. Bardella C, Costa B, Maggiora P, Patane S, Olivero M, Ranzani GN, De Bortoli M, Comoglio PM, Di Renzo MF. Truncated RON tyrosine kinase drives tumor cell progression and abrogates cell-cell adhesion through E-cadherin transcriptional repression. Cancer Res. 2004; 64:5154-5161.

20. Yokozaki H. Molecular characteristics of eight gastric cancer cell lines established in Japan. Pathol Int. 2000; 50:767-777.

21. Swanton C, Marani M, Pardo O, Warne PH, Kelly G, Sahai E, Elustondo F, Chang J, Temple J, Ahmed AA, Brenton JD, Downward J, Nicke B. Regulators of mitotic arrest and ceramide metabolism are determinants of sensitivity to paclitaxel and other chemotherapeutic drugs. Cancer Cell. 2007; 11:498-512.

22. Gavine PR, Mooney L, Kilgour E, Thomas AP, Al-Kadhimi K, Beck S, Rooney C, Coleman T, Baker D, Mellor MJ, Brooks AN, Klinowska T. AZD4547: an orally bioavailable, potent, and selective inhibitor of the fibroblast growth factor receptor tyrosine kinase family. Cancer Res. 2012; 72:2045-2056.

23. Moshitch-Moshkovitz S, Tsarfaty G, Kaufman DW, Stein GY, Shichrur K, Solomon E, Sigler RH, Resau JH, Vande Woude GF, Tsarfaty I. In vivo direct molecular imaging of early tumorigenesis and malignant progression induced by transgenic expression of GFP-Met. Neoplasia. 2006; 8:353-363.

24. Drebber U, Baldus SE, Nolden B, Grass G, Bollschweiler E, Dienes HP, Holscher AH, Monig SP. The overexpression of c-met as a prognostic indicator for gastric carcinoma compared to p53 and p21 nuclear accumulation. Oncol Rep. 2008; 19:1477-1483.

25. Takeuchi H, Bilchik A, Saha S, Turner R, Wiese D, Tanaka M, Kuo C, Wang HJ, Hoon DS. c-MET expression level in primary colon cancer: a predictor of tumor invasion and lymph node metastases. Clin Cancer Res. 2003; 9:1480-1488.

26. Sennino $B$, Ishiguro-Oonuma $T$, Schriver $B J$, Christensen JG, McDonald DM. Inhibition of c-Met reduces lymphatic metastasis in RIP-Tag2 transgenic mice. Cancer Res. 2013; 73:3692-3703.

27. Lee SJ, Seol HJ, Lee HW, Kang WY, Kang BG, Jin J, Jo MY, Jin Y, Lee J, Joo KM, Nam DH. Gene silencing of c-Met leads to brain metastasis inhibitory effects. Clin Exp Metastasis. 2013; 30:845-854.

28. Ponzetto C, Giordano S, Peverali F, Della Valle G, Abate ML, Vaula G, Comoglio PM. c-met is amplified but not mutated in a cell line with an activated met tyrosine kinase. Oncogene. 1991; 6:553-559.

29. Lutterbach B, Zeng Q, Davis LJ, Hatch H, Hang G, Kohl NE, Gibbs JB, Pan BS. Lung cancer cell lines harboring MET gene amplification are dependent on Met for growth and survival. Cancer Res. 2007; 67:2081-2088.

30. Zinser GM, Leonis MA, Toney K, Pathrose P, Thobe M, Kader SA, Peace BE, Beauman SR, Collins MH, Waltz SE. Mammary-specific Ron receptor overexpression induces highly metastatic mammary tumors associated with betacatenin activation. Cancer Res. 2006; 66:11967-11974.

31. Zhou D, Pan G, Zheng C, Zheng J, Yian L, Teng X. Expression of the RON receptor tyrosine kinase and its association with gastric carcinoma versus normal gastric tissues. BMC Cancer. 2008; 8:353.

32. Schroeder GM, An Y, Cai ZW, Chen XT, Clark C, Cornelius LA, Dai J, Gullo-Brown J, Gupta A, Henley B, Hunt JT, Jeyaseelan R, Kamath A, Kim K, Lippy J, Lombardo LJ, et al. Discovery of N-(4-(2-amino-3chloropyridin-4-yloxy)-3-fluorophenyl)-4-ethoxy-1-(4fluorophenyl)-2-oxo-1,2-dihydropyridine-3-carboxamide (BMS-777607), a selective and orally efficacious inhibitor of the Met kinase superfamily. J Med Chem. 2009; 52:1251-1254.

33. Benvenuti S, Lazzari L, Arnesano A, Li Chiavi G, Gentile A, Comoglio PM. Ron kinase transphosphorylation sustains MET oncogene addiction. Cancer Res. 2011; 71:1945-1955.

34. Ronsin C, Muscatelli F, Mattei MG, Breathnach R. A novel putative receptor protein tyrosine kinase of the met family. Oncogene. 1993; 8:1195-1202.

35. Angeloni D, Danilkovitch-Miagkova A, Ivanova $\mathrm{T}$, Braga E, Zabarovsky E, Lerman MI. Hypermethylation of Ron proximal promoter associates with lack of full-length Ron and transcription of oncogenic short-Ron from an internal promoter. Oncogene. 2007; 26:4499-4512.

36. Kawada I, Hasina R, Arif Q, Mueller J, Smithberger E, Husain AN, Vokes EE, Salgia R. Dramatic antitumor effects of the dual MET/RON small-molecule inhibitor LY2801653 in non-small cell lung cancer. Cancer Res. 2014; 74:884-895.

37. Lee NV, Lira ME, Pavlicek A, Ye J, Buckman D, Bagrodia S, Srinivasa SP, Zhao Y, Aparicio S, Rejto PA, Christensen JG, 
Ching KA. A novel SND1-BRAF fusion confers resistance to c-Met inhibitor PF-04217903 in GTL16 cells through [corrected] MAPK activation. PLoS One. 2012; 7:e39653.

38. Petti C, Picco G, Martelli ML, Trisolini E, Bucci E, Perera T, Isella C, Medico E. Truncated RAF kinases drive resistance to MET inhibition in MET-addicted cancer cells. Oncotarget. 2015; 6:221-233.

39. Zhang Z, Wang J, Ji D, Wang C, Liu R, Wu Z, Liu L, Zhu D, Chang J, Geng R, Xiong L, Fang Q, Li J. Functional genetic approach identifies MET, HER3, IGF1R, INSR pathways as determinants of lapatinib unresponsiveness in HER2-positive gastric cancer. Clin Cancer Res. 2014; 20:4559-4573.

40. Spigel DR, Ervin TJ, Ramlau RA, Daniel DB, Goldschmidt JH Jr., Blumenschein GR Jr., Krzakowski MJ,
Robinet G, Godbert B, Barlesi F, Govindan R, Patel T, Orlov SV, Wertheim MS, Yu W, Zha J, et al. Randomized phase II trial of Onartuzumab in combination with erlotinib in patients with advanced non-small-cell lung cancer. J Clin Oncol. 2013; 31:4105-4114.

41. Wu Z, Li J, Yang X, Wang Y, Yu Y, Ye J, Xu C, Qin W, Zhang Z. MCAM is a novel metastasis marker and regulates spreading, apoptosis and invasion of ovarian cancer cells. Tumour Biol. 2012; 33:1619-1628.

42. Schmittgen TD, Zakrajsek BA. Effect of experimental treatment on housekeeping gene expression: validation by real-time, quantitative RT-PCR. Journal of biochemical and biophysical methods. 2000; 46:69-81. 